\title{
Interoperabilidad digital en software educativo para la didáctica en la docencia universitaria
}

\section{Digital interoperability in educational software for didactics in university teaching}

\author{
Luis Javier Molina-Chalacan \\ p7001262479@ucvvirtual.edu.pe \\ Universidad Regional Autónoma de los Andes, Quevedo, Ecuador \\ Universidad César Vallejo, Trujillo, Perú \\ https://orcid.org/0000-0003-3755-2717
}

Recepción: 10 de mayo 2021

Revisado: 15 de junio 2021

Aprobación: 15 de agosto 2021

Publicación: 01 de septiembre de 2021 


\title{
RESUMEN
}

El docente, en los últimos años, ha tenido que incorporar a sus procesos didácticos distintos tipos de software educativos. En las universidades, pese a contar con capacitación sobre gestión de plataformas y herramientas digitales, no existe una dinámica que articule dichos recursos educativos con la didáctica del docente y las necesidades de aprendizaje del estudiante y promueva la tecnología de interoperabilidad digital; por consiguiente, cada vez es más necesario el desarrollo de competencias digitales por parte del docente universitario. Considerando esto, se presenta el artículo generado desde un proceso reflexivo con el propósito de mostrar la propuesta para el desarrollo de competencias en interoperabilidad digital (ID), diseñada para suscitar su fortalecimiento didáctico $\mathrm{y}$, por ende, del quehacer pedagógico de los estudiantes. Esto, a partir de la aplicación de conocimientos relacionados con los procesos didácticos mediados por las Tecnologías de la Información y las Comunicación (TIC) y la virtualización, que cada día es más evidente su uso.

Descriptores: Formación; ciencias de la educación; aplicación informática; información y comunicación. (Palabras tomadas del Tesauro UNESCO).

\begin{abstract}
In recent years, teachers have had to incorporate different types of educational software into their teaching processes. In universities, despite having training on the management of digital platforms and tools, there is no dynamic that articulates these educational resources with the teaching of the teacher and the learning needs of the student and promotes digital interoperability technology; consequently, the development of digital competences by university teachers is increasingly necessary. Considering this, the article generated from a reflexive is presented with the purpose of showing the proposal for the development of competencies in digital interoperability (ID), designed to provoke its didactic strengthening and, therefore, of the pedagogical work of the students. This, from the application of knowledge related to the didactic processes mediated by Information and Communication Technologies (ICT) and virtualization, whose use is more evident every day.
\end{abstract}

Descriptors: Training; educational sciences, computer applications, information and communication. (Words taken from the UNESCO Thesaurus). 
EPISTEME KOINONIA

Revista Electrónica de Ciencias de la Educación, Humanidades, Artes y Bellas Artes

Año IV. Vol IV. Nº. Julio - Diciembre. 2021

Hecho el depósito de Ley: FA2018000022

ISSN: 2665-0282

FUNDACIÓN KOINONIA (F.K).

Santa Ana de Coro, Venezuela

Luis Javier Molina-Chalacan

\section{INTRODUCCIÓN}

En el ámbito educativo universitario, las Tecnologías de la Información y la Comunicación (TIC), se han posicionado como instrumentos esenciales para el tratamiento de la información que permiten el enriquecimiento de los conocimientos y habilidades técnicas. No obstante, estos nuevos contextos de relación educacióntecnología impulsa el repensamiento de las formas y maneras didácticas de enseñar y aprender (Sunkel, Trucco y Espejo, 2013), lo que genera, consecuentemente, nuevos desafíos. Sobre todo, en estos momentos cuando gran parte del mundo vive un estado de confinamiento debido a la pandemia causada por el virus de Covid19.

En tal sentido, emerge el reto de la interoperabilidad digital (ID) en la educación, que no es más que lograr que los distintos sistemas, aplicaciones y datos que contienen, se interconecten de manera eficiente dentro de la organización educativa. Es decir, refiere a la forma como varios componentes digitales heterogéneos o programas de diferentes plataformas, que se usan en una aula digital o presencial, logran interactuar fluidamente sin inconvenientes en el proceso, generando que los estudiantes puedan acceder a un conjunto de herramientas que les permita vivir y experienciar un proceso de educativo y de aprendizaje de calidad, al mismo tiempo que les permite a las instituciones educativas compartir y gestionar fácilmente los sistemas de datos (Open Assessment Technologies, 2019).

Es por ello que, en la didáctica del docente universitario, cada vez es más necesario que se incluya competencias digitales para la producción de materiales educativos heterogéneos y, así, poder lograr interoperabilidad digital en el aula, sea esta física o virtual. Pues, a pesar de que la universidad capacita al docente en el uso de herramientas digitales, la gestión de plataformas educativas y otros aspectos sobre teorías de aprendizaje, estos procesos no se concretan, ya que, no se forja en él, un real proceso de producción de sus propios materiales digitales fortalecedores de los procesos didácticos, ni mucho menos, existe una dinámica que acople estos aspectos y promueva la tecnología de interoperabilidad digital. 


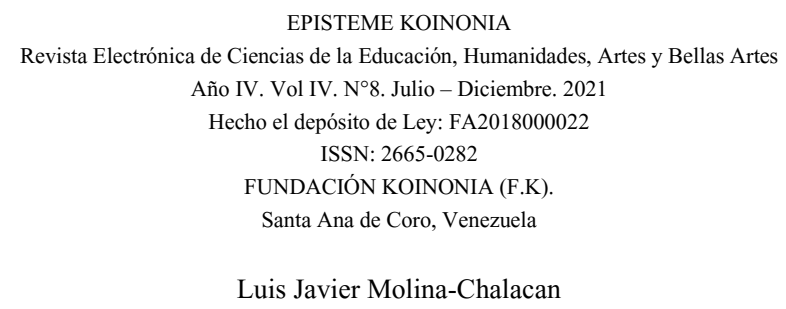

Considerando lo planteado, se presenta el siguiente artículo de reflexión que muestra la propuesta para el desarrollo de competencias en ID, diseñada como parte de un proceso que ha de suscitar el fortalecimiento didáctico de los docentes universitarios $y$, por ende, del quehacer pedagógico de los estudiantes. Pues, en la actualidad, y debido a la tecnología, el aprendizaje ha cambiado, por lo tanto, la forma de enseñar y de aprender se han de transformar (Downes, 2014).

De allí que, el trabajo, metodológicamente se desarrolla a partir de un proceso reflexivo que pone en evidencia la necesidad de desarrollar en los docentes, competencias en ID de Software Educativo desde el enfoque conectivista. Concretamente, el artículo se ha estructurado en tres partes: la primera parte, refiere a los argumentos teóricos conceptuales como la teoría Conectivista de Siemens, la IO en software educativo y la didáctica universitaria, los cuales son fundamentales para el desarrollo de la propuesta. La segunda parte, alude al modelo diseñado como propuesta para el impulso de competencias en ID de Software Educativo y, la tercera parte, alude a los aportes generados del proceso reflexivo.

\section{ARGUMENTOS TEÓRICOS CONCEPTUALES}

Para desarrollar el proceso de fortalecimiento didáctico del docente universitario en el ámbito de las TIC, se hace necesario esbozar algunos argumentos referenciales que permiten situar el proceso de ID de software educativo, en el contexto una didáctica que se ha de apropiar de la teoría conectivista como enfoque pedagógico. En tal sentido, estos argumentos son:

\section{La didáctica universitaria en el ámbito de las TIC}

En la actualidad, el proceso educativo universitario debe responder a las demandas sociales que requiere la sociedad; debido a ello, las organizaciones universitarias están obligadas a sumar esfuerzos para perpetrar grandes cambios en los modelos educativos, sobre todo, en vista de la necesidad generar cambios culturales, con los diversos actores universitarios, para incorporar las TIC a la acción educativa. Pues, los procesos de globalización y de desarrollo tecnológico exigen de la universidad, la 
EPISTEME KOINONIA

recreación constante de sus modos y formas de generación de conocimientos acordes a la realidad (Silva, 2019). Con ello, la didáctica asume un rol primordial, pues, es una disciplina pedagógica fundamental para el desarrollo de los procesos formativos y la gestión de la enseñanza y el aprendizaje, que se ha de interrelacionar con las TIC. Etimológicamente, la palabra didáctica, que deriva del griego, está conformada por los vocablos "didaskein" que significa enseñar y "tekne" que se traduce como arte o técnica; por consiguiente, alude a ciencia y arte de enseñar (Diccionario etimológico dechile.net.) La didáctica como proceso relativo a la enseñanza, se constituye en una disciplina emergente que se inscribe en las ciencias educativas. Su quehacer, desde lo inscrito por Buitrago (2008), se enmarca en el abordaje de las prácticas pedagógicas que subyacen al encuentro educativo y los fundamentos epistemológicos que los actores educativos ponen en juego en el acto didáctico mismo. De allí que, tiene como punto nuclear al propio proceso de enseñanza-aprendizaje, a fin de optimizar los métodos, técnicas y herramientas pedagógicas implicados en él.

La didáctica, a pesar de ser una disciplina joven, en su corta historia ha evolucionado, en este sentido y considerando lo inscrito por Gonzáles y Castrillón (2001), referenciados por Buitrago (2008), la significación más tradicional la refiere como la técnica que le permite al docente transmitir saberes, de manera coherente y organizada, a fin de que los estudiantes desarrollen aprendizajes en situaciones concretas.

No obstante, con el transcurrir del tiempo, situaciones como: la necesidad de acercar el saber científico y el saber pedagógico, el lenguaje científico con el lenguaje cotidiano; la emergencia de la TIC como instrumentos mediadores del proceso de enseñanza-aprendizaje, han conllevado a que la didáctica se resignifique ahora "como una de las ciencias de la educación en pleno desarrollo" (Abreu, Gallegos, Jácome y Martínez, 2017; p. 89). Pues, gracias a ella, los docentes pueden diseñar las situaciones de enseñanza-aprendizaje y desplegar las estrategias formativas que permiten, no solo un mejor aprendizaje, sino, también la capacidad de poder desaprender y reaprender de manera continua y a lo largo de la vida (Sito, 2019) 


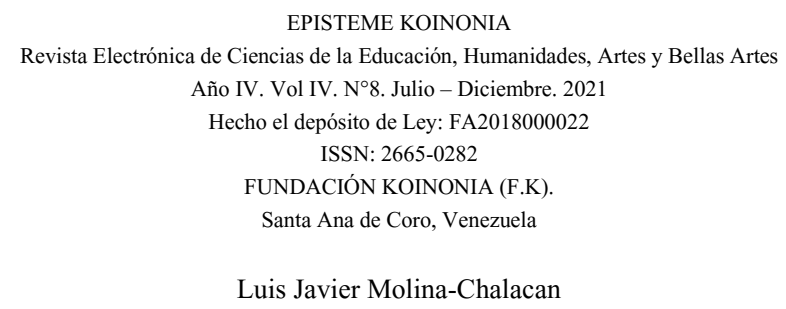

Ahora bien, en lo que concierne a la didáctica universitaria, es de saber que esta se constituye en un núcleo disciplinar, dentro de la didáctica general, que se diferencia de otras didácticas específicas. Significa entonces, que ella se puede conceptuar como la esfera del conocimiento que se ocupa y trata los métodos, técnicas y herramientas pedagógicas implicados en el proceso de enseñanza-aprendizaje en el contexto universitario; es decir, es una didáctica propia del mundo de la universidad. En relación a esto último y considerando lo expresado por Silva (2019), la universidad hoy día requiere desarrollar una didáctica mucho más real y certera, pues, el proceso de enseñanza-aprendizaje se apoya en las TIC y los espacios formativos ahora se estructuran y organizan teniendo como sostén fundamental a los entornos virtuales y las tecnologías ubicuas, los cuales, permiten la conectividad e interconexión para el aprendizaje y la gestión del conocimiento en cualquier lugar y momento. En concreto, estos nuevos paradigmas del aprendizaje y el conocimiento son determinantes en los cambios que ha de dar la universidad, en cuanto a una didáctica innovadora que muestre el encuentro entre la educación y las TIC (Ortiz y Martínez, 2017).

\section{Teoría Conectivista de Siemens}

En el contexto de desarrollo de una didáctica universitaria mediada por la TIC es menester mostrar una teoría fundamentadora del proceso de aprendizaje. En tal sentido, el Conectivismo se constituye epistemológicamente en un enfoque que busca comprender y explicar cómo es que la persona aprende de manera compleja y crea conocimiento en una sociedad digitalizada en rápida evolución (Area, 2014 y Siemens, 2004).

El Conectivismo postula, esencialmente, que el aprendizaje y conocimiento ya no son una actividad puramente interna e individual del ser humano, pues, se da con las interconexiones que ocurren dentro de las redes. Es decir, aunque tienen como punto de partida al individuo, son producto de las conexiones y las conectividades que suceden entre todos los nodos en una red (social, neuronal). Entendiendo que un nodo puede ser cualquier cosa que provee información, como una organización, datos, sentimientos e imágenes (Bates, 2019; González, 2017 y Area, 2014). 
EPISTEME KOINONIA

Considerando lo expuesto y concatenándolo con lo enunciado por Siemens (2004), se puede decir que el aprendizaje y el conocimiento, penden de las conexiones y de la forma de fluir de las informaciones. En tal sentido, el docente, en esta era de la educación mediada por lo digital, debe coadyuvar a la creación de entornos personales de aprendizaje que permitan el afloramiento de conexiones a redes exitosas. Claro está, considerando tanto que las TIC son instrumentos fundamentales para el aprendizaje y el trabajo de enseñanza virtual; así como, que el conocimiento no solo está en las personas y organizaciones, sino, también, en los repositorios de información, las plataformas virtuales, las redes sociales, las múltiples aplicaciones y recursos de la Web como: blogs, wikis, motores de búsquedas web, webquest, los entornos para compartir recursos (Google Drive, Podcast, Vodcast, Youtube, Instagram, Ustream, Dropbox), entre otros (González, 2017).

Cabe resalta que, aunque la teoría conectivista "está más enfocada en los participantes individuales, las redes, el flujo de información y las nuevas formas de conocimiento resultantes" (Bates, 2019, p.s/p), el docente tiene el rol fundamental para la creación de "ecologías de aprendizajes" (Gutiérrez, 2012), en contraposición a la función de planear diseños instruccionales didácticamente estáticos. Estas ecologías de aprendizajes, como expresan Martinenco, Martín y García (2021) desde lo inscrito por Barron (2006), se constituyen en entidades dinámicas que se estructuran combinando e integrando contextos formales, no formales e informales para el aprendizaje; donde, cada uno de estos contextos ha de contener actividades, recursos, relaciones e interacciones esenciales a ellos.

En concreto, la creación de estas ecologías orientadas al desarrollo de aprendizajes desde la hibridación de contextos en los procesos de educación virtual, asume como estrategia de funcionamiento la creación de bloques de repositorios y el uso de redes, plataformas, aplicaciones y múltiples recursos de la Web, donde una de entre las múltiples características ha de ser la interoperabilidad de ellos (Nieto, 2009). Entendiendo está interoperabilidad como la cualidad que expresa la capacidad de los sistemas informáticos, aplicaciones y servicios, de funcionar e integrarse de forma correcta con otros sistemas, a la vez que comunican, comparten e intercambian datos, 


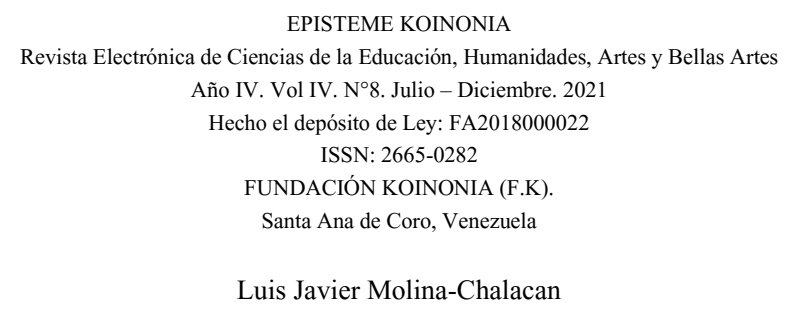

y posibilitan informaciones y conocimientos de manera precisa, efectiva y permanente entre sí (Martínez y Lara, 2006).

\section{La interoperabilidad digital en Software Educativo}

Para el buen desarrollo de un proceso didáctico mediado por las TIC y estructurados con software educativos o aplicaciones y recursos educativos digitales que faciliten el proceso de enseñanza aprendizaje (Vidal, Gómez y Ruiz, 2010), la interoperabilidad de los sistemas que lo soporten se constituye en un elemento fundamental para ello. En tal sentido, el vocablo Interoperabilidad procede de la palabra anglosajona interoperability que traducido al español ha originado dos posibles alternativas interoperatividad o interoperabilidad; no obstante, hoy día se opta por el termino interoperabilidad (Correa, Di Petta y Frugoni, 2011). Asimismo, es de saber que la expresión "interoperable" se constituye a partir de sus raíces etimológicas latinas inter que significa "entre" y operable que se entiende como "que pueden trabajar junto entre"

Para entender mejor lo que es la interoperabilidad es preciso traer a colación algunas definiciones establecidas. En este tenor, el Institute of Electrical and Electronics Engineers, referenciado por la OPS/OMS (2016), expresa que la interoperabilidad es la habilidad o capacidad de dos o más sistemas de intercambiar información y utilizar la información intercambiada. De igual forma, Gradman (2009) citado por Nogales (2019) la refiere como "una característica esencial para arquitecturas de información enlazadas para trabajar en entornos con parámetros heterogéneos y a lo largo del tiempo" (p.6).

Según González (2013), la interoperabilidad en el contexto de las TIC, es la habilidad para transferir y traducir datos útiles y otra clase de información entre sistemas operativos, aplicaciones o componentes. Asimismo, la Norma Internacional ISO19101:2002, citada por Manso, Wachowicz, Bernabé, Sánchez y Rodríguez (2008), precisa la interoperabilidad como la capacidad que tienen los sistemas y/o sus componentes de realizar procesos de intercambio de información, además de, reconocer y controlar dicho proceso cooperativo. 
Considerando estas definiciones, se puede deducir que la interoperabilidad revela la correcta interconexión que se puede dar entre dos o más sistemas y/o software para que trabajen juntos. Asimismo, esta interconexión se caracteriza por reconocer y controlar los procesos cooperativos de enlace, intercambio, traducción y utilización de datos e informaciones, con los que se pueden organizar entornos híbridos de trabajo, en este caso entornos educativos y de aprendizaje.

Cabe agregar que, para lograr efectivamente la interoperabilidad entre los sistemas y/o software, en este caso educativos y de aprendizaje, esta se debe dar en distintos niveles. En tal sentido y considerando lo expuesto por Manso, Wachowicz, Bernabé, Sánchez y Rodríguez (2008); Martínez y Lara (2007) y Gómez (2007), una clasificación aplicable comprende los niveles de interoperabilidad técnica, sintáctica, semántica y organizacional, tal como se muestra en la siguiente figura:

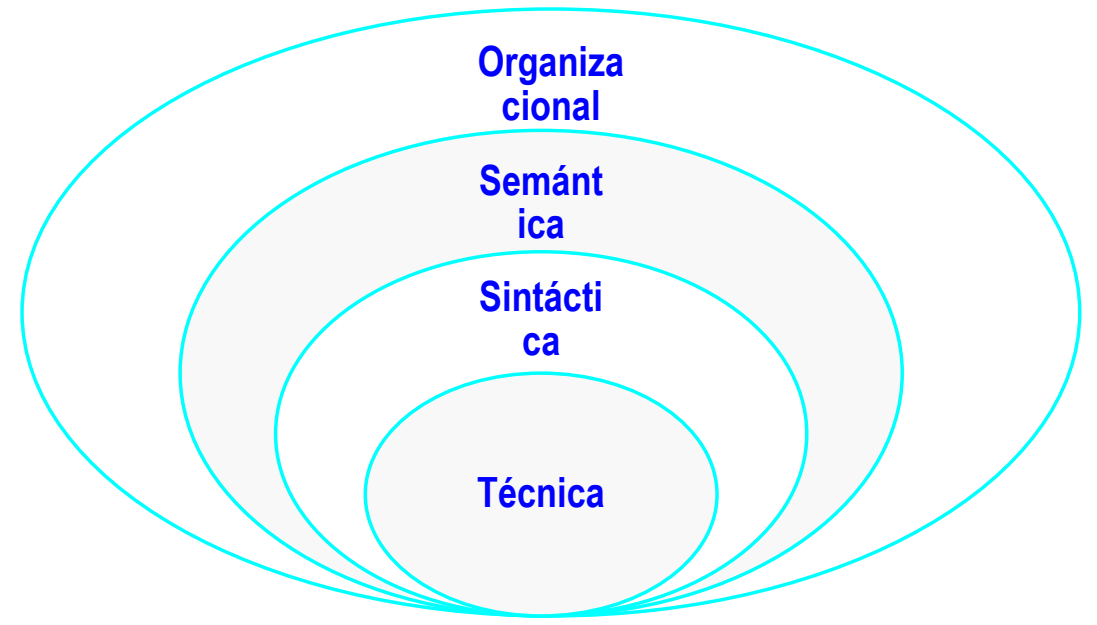

Figura 1. Niveles de Interoperabilidad

Fuente: El autor. 


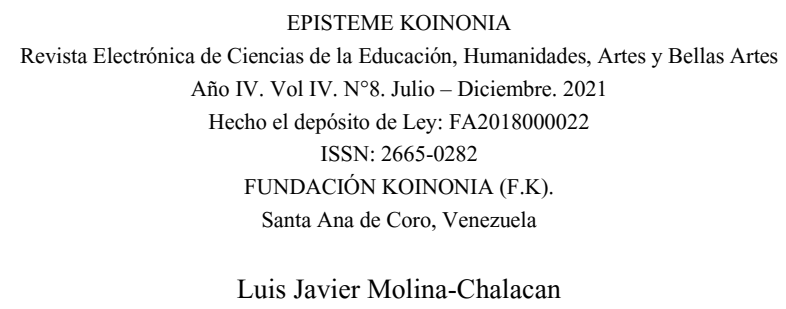

De manera concisa, la interoperabilidad técnica refiere a los protocolos, códigos informáticos e infraestructura (hardware y/o software, sistemas y plataformas), que permiten la interconexión de máquina a máquina. Asimismo, la interoperabilidad sintáctica, tiene que ver con la estandarización de formatos que posibilita el intercambio de información. En cuanto a la interoperabilidad semántica, esta se relaciona con el uso de un vocabulario común y compartido que permite intercambio de información y posibilita el descifrado y entendimiento de los significados del contenido por ambos sistemas. Por último, la interoperabilidad organizacional, la cual posibilita el conocimiento y discernimiento en torno a modelos de procesos, políticas de acceso, uso de datos y/o servicios, objetivos y fines de la organización, al crear esta misma un dato o proporcionar un servicio, en este caso educativo.

Para finalizar, los distintos niveles de interoperabilidad que se han de desarrollar en las organizaciones, aunque, como expresa González (2013), "pueden verse reflejadas en distintas iniciativas electrónicas de envergadura como: el comercio electrónico; los servicios prestados por las Administraciones Públicas, como servicios sanitarios, de justicia, de administración electrónica" (p.26); en los escenarios educativos que están arropados de iniciativas e-learning o procesos de enseñanza-aprendizaje mediados por la internet y las TIC, tienen presencia y revisten de gran importancia. Pues, en esta era del conocimiento y la digitalización, representan una de las mejores vías que posibilita los cambios que se están generando en los modos y maneras de formación humana. Aunado a que la tecnología y su funcionalidad han de ser garantes de procesos que apunten a la formación integral de las personas, las cuales, debe responder a las demandas de la realidad social de manera eficiente (Vidal, Rodríguez y Martínez, 2014). 


\section{PROPUESTA PARA LA FORMACIÓN DOCENTE EN INTEROPERABILIDAD DIGITAL DE SOFTWARE EDUCATIVO}

Hoy día, cuando es ineludible para la universidad configurar sus procesos educativos y de enseñanza-aprendizaje mediante un uso efectivo de las TIC, es apremiante para ella hacer una profunda revisión de sus enfoques y estrategias de gestión pedagógica y de capacitación docente respecto a ello. En tal sentido, se presenta el modelo de capacitación denomina "Formación Docente en Interoperabilidad Digital de Software Educativo", con el propósito de fortalecer la didáctica del docente universitario, ahora mediadas por el uso de las TIC y la virtualidad.

Dicho modelo se diseñó teniendo como sustento pedagógico la teoría Conectivista de Siemens que, dentro de sus principios más representativo, expone que el aprendizaje inicia con el individuo, en este caso al estudiante, sin embargo, ya no se pueden asumir como una acción únicamente personal e individualista; pues, como expresa Downes (2007) citado por Bates (2019), el conocimiento se estructura y distribuye a través de redes de conexiones, de allí que, el aprendizaje se ha de mostrar como la capacidad de construir formas y atravesar esas redes.

De igual forma, expresa que el conocimiento puede residir en mecanismos no humanos; esta parte es esencial, puesto que, en la propuesta, estos dispositivos mencionados son los softwares educativos, que contienen una vasta información de contenidos, estrategias propias del docente y material digitales; los cuales, requieren sean interoperables o trabajen de manera efectiva entre sí. Pues, todo esto contribuyen a estar conectados en redes y facilitar el aprendizaje que se da en ambientes difusos y con elementos cambiantes (Siemens, 2004).

Cabe resaltar que en la esfera de la ID interoperabilidad y para el desarrollo de software educativo, se analizaron algunas métodos como: MERISE, Adaptative Software Development (ASD), Dynamic System Develop Method (DSDM), FeatureDriven Development (FDD), Programación Extrema XP, Modelo Sistémico de Calidad (MOSCA) del Software, Metodología Dinámica para el desarrollo de Software Educativo, Metodología de Desarrollo de Software Educativo una propuesta metodológica y MODESEC (Dueñas, Gómez, Toscana y Caro, 2017). Con esta 
revisión se pudo estructurar una metodología que facilita la construcción de la ID de Softwares Educativos; dicha metodología se ha denominado con el acrónimo de IDADICOR-SE y se estructura con el desarrollo de cuatro etapas indicadas como: Análisis, Diseño, Construcción, y Retroalimentación/Implementación, tal como se muestra en la siguiente figura:

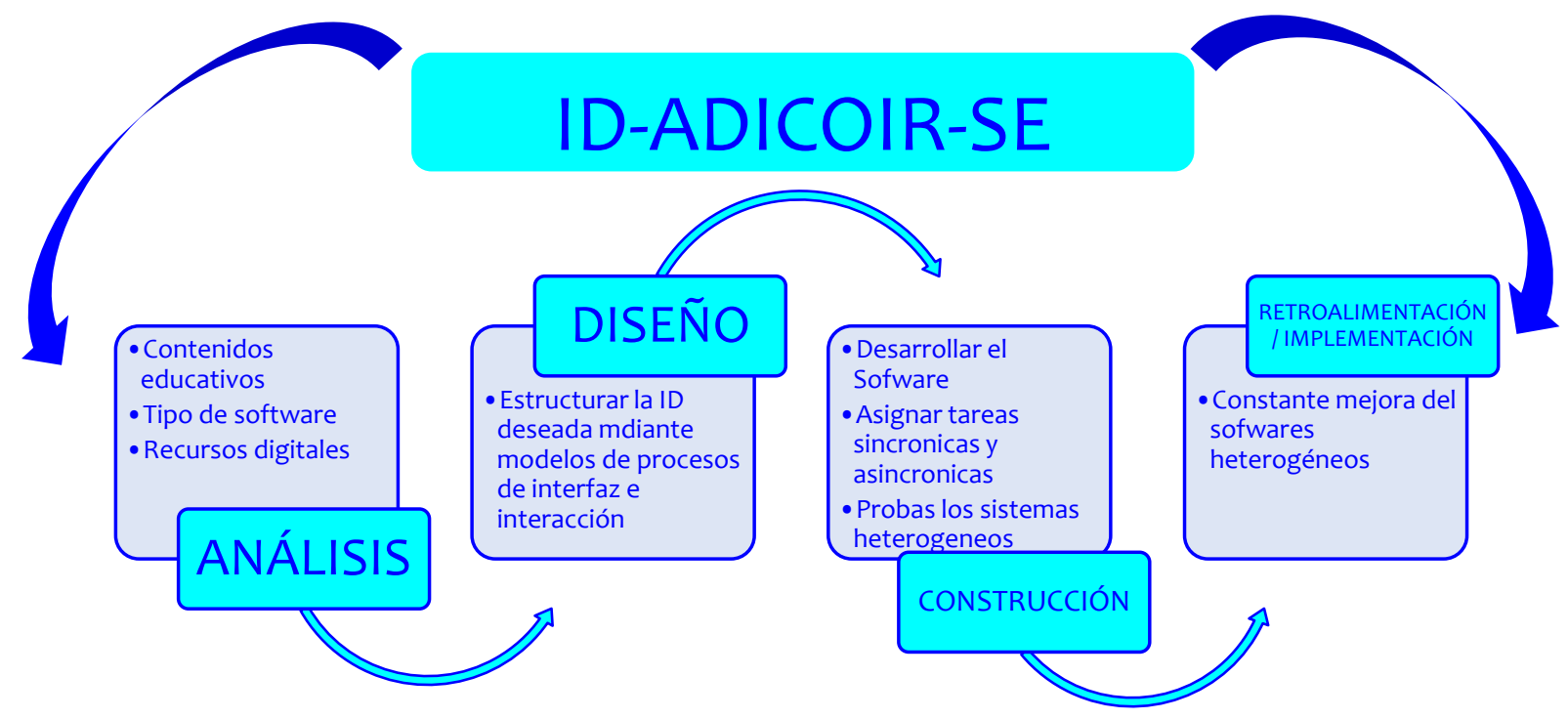

Figura 2. Metodología para el desarrollo de la ID en Softwares Educativos. Fuente: El autor.

De manera sintética, el modelo de formación se ha configurado considerando lo siguiente: 


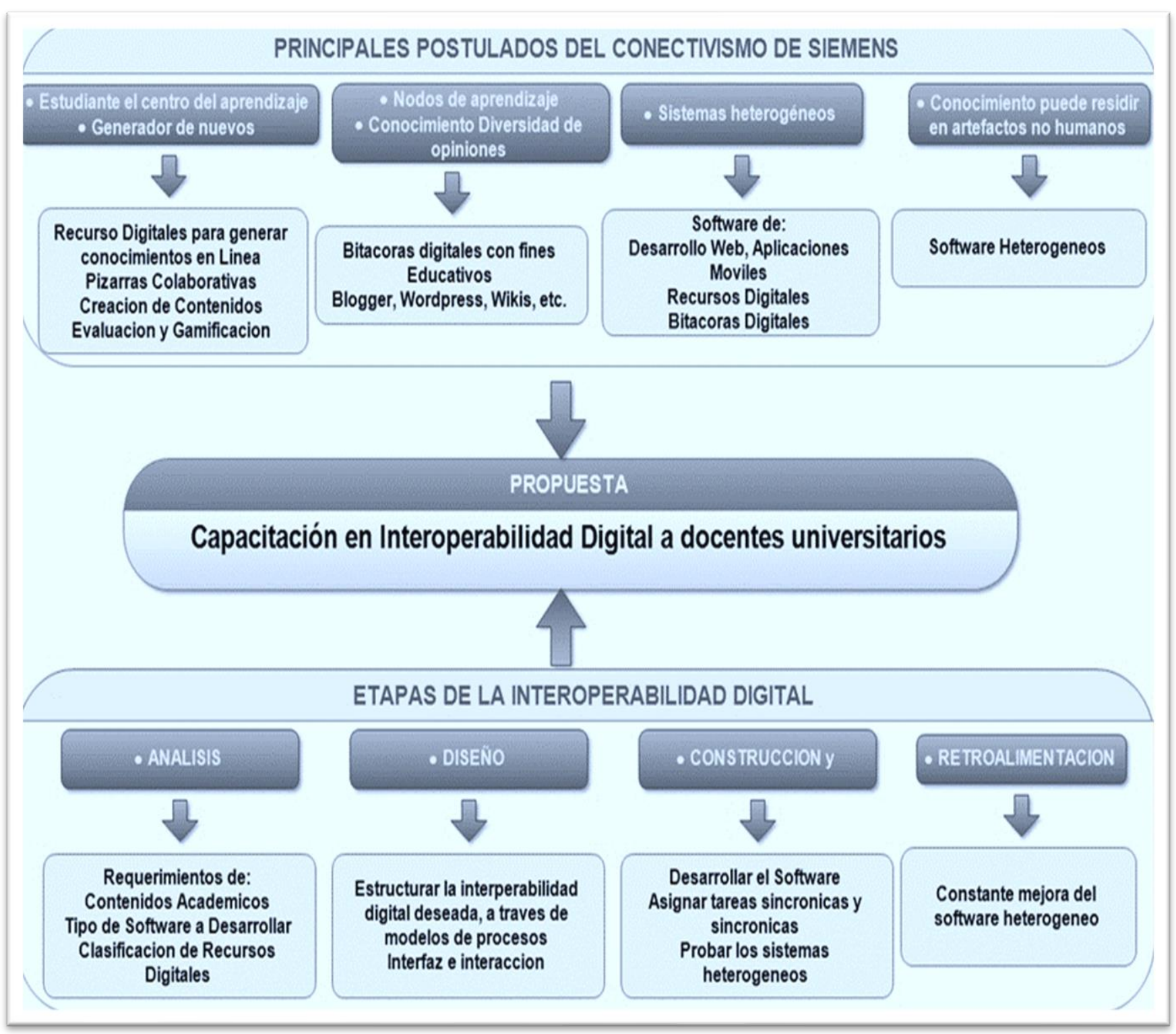

Figura 3. Elementos configuradores de la propuesta Fuente: El autor. 
De igual forma, el diseño del modelo se ha dispuesto siguiendo la lógica de planificación de fases y sesiones, de acuerdo con lo que implica un trabajo propositivo. En tal sentido, se estructuraron cuatro fases, tal como se muestra en la siguiente figura:

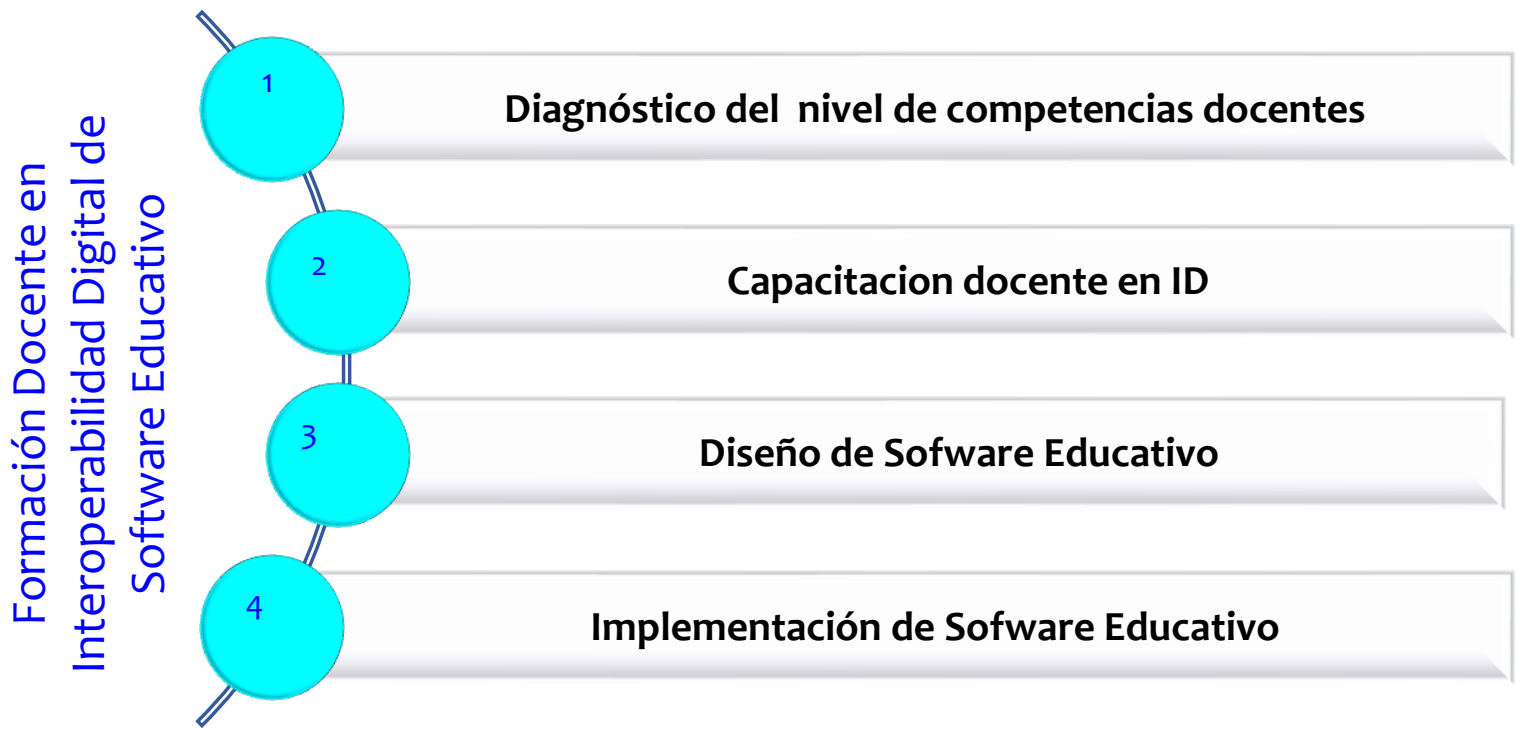

Figura 4. Lógica de planificación de fases.

Fuente: El autor.

La primera fase, implica la socialización de la propuesta. De igual manera involucra la realización de un diagnóstico de la situación actual de las competencias docentes en didáctica virtual y, el desarrollo y manejo de software heterogéneo. Para ello se requiere determinar y aplicar técnicas e instrumentos de evaluación; asimismo, para se sugiere la utilización de programas y herramientas tecnológicas para la tabulación y análisis e interpretación de los datos. 
La segunda fase, se estructura mediante 9 encuentros didácticos para la capacitación en ID dirigido a docentes universitarios. En tal sentido, se presenta la planificación diseñada para tal fin, especificando los objetivos y contenidos temáticos por cada encuentro:

\section{Cuadro 1.}

Plan de encuentros didácticos.

\begin{tabular}{|c|c|c|}
\hline ENCUENTRO & OBJETIVO & CONTENIDO TEMÁTICO \\
\hline 1 & Definir conceptos básicos & $\begin{array}{ll}\text { - } & \text { Interoperabilidad Digital } \\
\text { - } & \text { El software educativo (SE), tipos } \\
\text { Estructura, Utilización } & \text { e } \\
\text { Importancia }\end{array}$ \\
\hline 2 & $\begin{array}{l}\text { Analizar los postulados del } \\
\text { conectivismo y sus aportes a la } \\
\text { propuesta }\end{array}$ & $\begin{array}{l}\text { - El conectivismo, postulados y su } \\
\text { relación con la propuesta } \\
\text { - Estructura relacional, Software } \\
\text { Heterogéneo, Nodos de } \\
\text { Aprendizaje, Almacenamiento del } \\
\text { conocimiento, Plataformas de } \\
\text { distribución }\end{array}$ \\
\hline 3 & $\begin{array}{l}\text { Analizar diversos Modelos de } \\
\text { Desarrollo Software Educativo }\end{array}$ & $\begin{array}{l}\text { - } \quad \text { Modelos de desarrollo Software } \\
\text { - } \quad \text { Frameativo } \\
\text { - } \quad \text { Modelo Desarrollo Web } \\
\text { - } \quad \text { Modelo Desarrollo Móvil }\end{array}$ \\
\hline 4 & $\begin{array}{l}\text { Diseñar y socializar el modelo de } \\
\text { desarrollo de software Educativo }\end{array}$ & $\begin{array}{l}\text { - Descripción de las etapas de la } \\
\text { propuesta, valoración Inicial } \\
\text { - Diseño Didáctico, Tipo de } \\
\text { Desarrollo, Retroalimentación } \\
\text { - Aplicación }\end{array}$ \\
\hline 5 & $\begin{array}{l}\text { Describir y practicar el uso de las } \\
\text { pizarras colaborativas }\end{array}$ & $\begin{array}{l}\text { Ingreso, uso, compartir, exportar } \\
\text { en: Miro www.miro.com } \\
\text { - Padlet www.es.padlet.com, Jam } \\
\text { board www.edu.google.com. }\end{array}$ \\
\hline 6 & $\begin{array}{l}\text { Describir y practicar el uso de } \\
\text { organizadores de ideas }\end{array}$ & $\begin{array}{l}\text { Ingreso, uso, compartir, exportar } \\
\text { en: } \\
\text { Canva www.canva.com, Genially } \\
\text { www.genial.ly, } \\
\begin{array}{ll}\text { www.goconqr.com, } \\
\text { www.visme.co }\end{array}\end{array}$ \\
\hline 7 & $\begin{array}{l}\text { Describir y practicar el uso de } \\
\text { Bitácoras Digitales (Blogs) }\end{array}$ & $\begin{array}{l}\text { Ingreso, uso, compartir, exportar } \\
\text { en: Blogger www.blogger.com, } \\
\text { Wordpress www.wordpress.com. }\end{array}$ \\
\hline 8 & $\begin{array}{l}\text { Describir y practicar el uso de } \\
\text { aplicaciones para evaluación y } \\
\text { gamificación }\end{array}$ & $\begin{array}{l}\text { - Ingreso, uso, compartir, exportar } \\
\text { en: Google Forms } \\
\text { www.docs.google.com, Quizizz. }\end{array}$ \\
\hline
\end{tabular}




\begin{tabular}{l|l|l}
\hline & & \multicolumn{2}{c}{$\begin{array}{l}\text { www.quizizz.com, Kahoot } \\
\text { www.kahoot.com }\end{array}$} \\
\hline 9 & $\begin{array}{l}\text { Describir y practicar el uso de } \\
\text { aplicaciones para Evaluación y y } \\
\text { gamificación }\end{array}$ & $\begin{array}{l}\text { Ingreso, uso, compartir, exportar } \\
\text { en: Wall Word } \\
\text { www.wordwall.net/es, Ardora } \\
\text { www.webardora.net }\end{array}$ \\
\hline
\end{tabular}

Fuente: El autor.

La tercera fase, implica el desarrollo del de Software Educativo siguiendo la metodología ID-ADICOR-SE, ideada para ello. En esta fase se sugiere el uso de Entornos de Desarrollo Integrados (IDE), en desarrollo web Adobe Dreamweaver y en móvil Android Studio, considerando sus ventajas de uso, flexibilidad de código entre otros aspectos. Así mismo se sugiere el uso de varias herramientas digitales como: pizarras colaborativas (Miro, Padlet, Jamboard), creación de contenidos organizadores de ideas (Canva, Genially, Gocongr, Visme), bitácoras digitales (Blogger, Wordpress), evaluación y gamificación (Quizizz, Kahoot.It, Word Wall, Ardora).

Estas herramientas han sido seleccionadas tras un análisis de varias características como: Interfaz amigable, suficientes herramientas habilitadas para el modo gratuito, fácil uso por parte de docentes y estudiantes, frecuencia y número de usuarios, calidad de herramientas para la producción y aprendizaje, número de plantillas y herramientas disponibles, exportación a formatos PDF o JPG y la rapidez de carga de las aplicaciones en línea. Considerando esto, a continuación, se detalla cada etapa de la metodología ID-ADICOR-SE, así como sus respectivas actividades: 


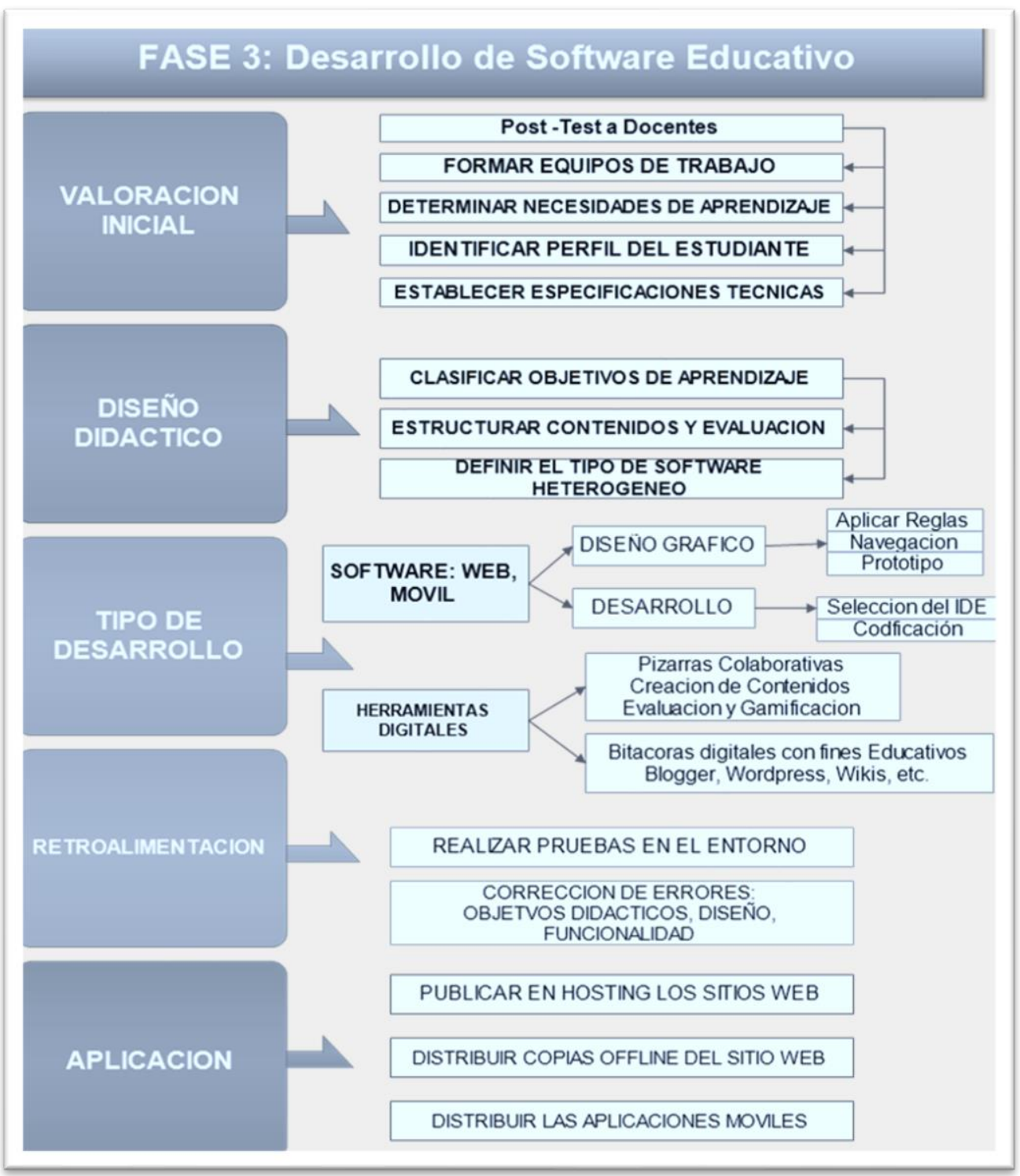

Figura 5. Etapas y actividades de la metodología ID-ADICOR-SE para el desarrollo de Software Educativo

Fuente: Los autores. 
Cabe agregar que, para lograr el desarrollo de software educativo heterogéneo, es necesario cumplir con siete sesiones de trabajo tipo taller; por lo que a continuación, se detalla cada actividad:

\section{Cuadro 2.}

Plan de encuentros didácticos.

\begin{tabular}{|c|c|c|}
\hline TALLER & OBJETIVO & ACTIVIDADES \\
\hline 1 & $\begin{array}{l}\text { Analizar la situación } \\
\text { inicial }\end{array}$ & $\begin{array}{l}\text { - } \text { Aplicar instrumentos de evaluación } \\
\text { - } \text { Formar equipos de trabajo } \\
\text { - } \text { Determinar necesidades de aprendizaje } \\
\text { - } \text { Identificar perfil del estudiante } \\
\text { - Establecer especificaciones técnicas }\end{array}$ \\
\hline 2 & $\begin{array}{l}\begin{array}{l}\text { Elaborar el diseño } \\
\text { didáctico }\end{array} \\
\end{array}$ & $\begin{array}{l}\text { - } \quad \text { Clasificar objetivos de aprendizaje } \\
\text { - } \quad \text { Estructurar contenidos y evaluación } \\
\text { - } \quad \text { Definir el tipo de software heterogéneo }\end{array}$ \\
\hline 3 & $\begin{array}{l}\text { Desarrollar prototipos } \\
\text { de aplicaciones móviles } \\
\text { educativas }\end{array}$ & $\begin{array}{l}\text { - } \quad \text { Diseño de prototipo } \\
\text { - } \quad \text { Diseño de interfaz } \\
\text { - } \quad \text { Estructura del prototipo } \\
\text { - } \quad \text { Desarrollo por especialistas }\end{array}$ \\
\hline 4 & $\begin{array}{l}\text { Desarrollar prototipos } \\
\text { de aplicaciones web. }\end{array}$ & $\begin{array}{l}\text { - } \text { Diseño de prototipo } \\
\text { - } \text { Diseño de interfaz } \\
\text { - Estructura del prototipo } \\
\text { - } \text { Desarrollo por especialistas }\end{array}$ \\
\hline 5 & $\begin{array}{l}\text { Desarrollar } \quad \text { actividades } \\
\text { sincrónicas } \\
\text { asincrónicas } \quad \text { ytilizando } \\
\text { recursos digitales }\end{array}$ & $\begin{array}{l}\text { - Clasificar de actividades sincrónicas y } \\
\text { - Sincrónicas } \\
\text { - Sistematizar actividades con recursos } \\
\text { - Sistizar evaluación con recursos }\end{array}$ \\
\hline 6 & $\begin{array}{l}\text { Determinar posibles } \\
\text { fallos y corregir }\end{array}$ & $\begin{array}{l}\text { - Realizar pruebas del software heterogéneo } \\
\text { - Corregir errores en contenidos, código, } \\
\text { diseño y otros }\end{array}$ \\
\hline
\end{tabular}

Fuente: Los autores.

Con la cuarta fase, se busca implementar el software educativo heterogéneo, en la comunidad educativa, para lo cual se recurre a repositorios en la nube para su fácil propagación. Además, las herramientas digitales como pizarras colaborativas, creación de contenidos, bitácoras, evaluación y gamificación, se las puede distribuir a 


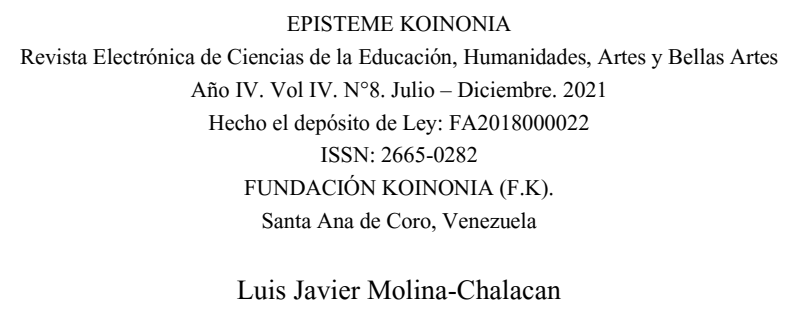

través de plataformas tipo Moodle que tenga la institución, tanto para tareas sincrónicas, como asincrónicas.

\section{APORTES REFLEXIVOS}

En la actualidad producto del confinamiento debido al Covid19, se ha dispuesto las clases online. Tal situación, inmediatamente, ha producido la necesidad urgente de aplicar procesos didácticos virtuales mediante el uso de software educativo; de allí que, es menester fortalecer las practicas pedagógicas del docente como mediador virtual. Sin embargo, a pesar de que se ha dado capacitaciones en manejo de herramientas digitales, se observa la poca interoperabilidad entre los elementos de la clase, la didáctica del docente, los contenidos curriculares con sus logros de aprendizajes y la herramienta digital adecuada, que logre sistematizar los aprendizajes.

Una de las soluciones para que estos procesos didácticos virtuales se fortalezcan, es tener claro lo que representa la teoría conectivista en virtud de asumir que el aprendizaje, para generar sujetos independientes y auto organizados productores de conocimientos, implica crear y usar redes con las que se pueda estructurar nodos e interconexión eficaces, a través de aplicaciones y recursos educativos digitales. Para ello es menester aplicar un modelo sistémico de interoperabilidad digital en software educativos. En este sentido, la interoperabilidad digital dinamiza los procesos entre software heterogéneos; al mismo tiempo, que facilita la interacción entre el docente, el software heterogéneo y los estudiantes. No obstante, esta sinergia se logra cuando el docente universitario mejora sus competencias digitales, a través de necesarias capacitaciones para ello.

De allí que, la propuesta de formación en ID de Software Educativo se presenta como una opción que posibilita el fortalecimiento didáctico en docentes universitarios, al crear un producto tangible como requerimiento y su aplicación, que en este caso es el software educativo heterogéneo y personalizado.

El software que se origine bajo los requerimientos académicos-didácticos y heterogéneos, se pueden encontrar en varios sitios web de distinto manejo y 


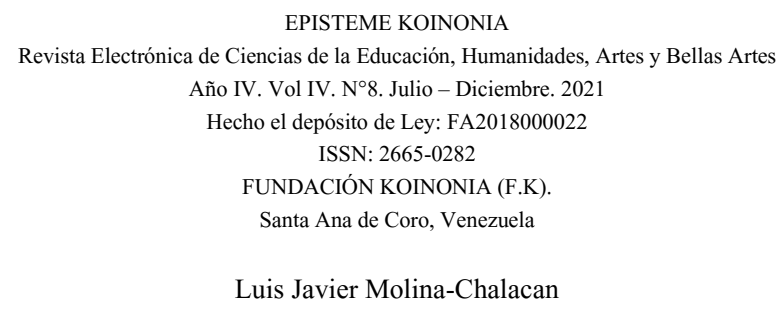

configuración para generar conocimientos e internaciones en línea, como por ejemplo Canva, Jamboard, Miro, entre otros; así como también pueden estar en software educativos para escritorios, los mismos que se podrán distribuir a través de almacenamientos en la nube, podrán ser descargados e instalados en equipos tanto móviles como de escritorio, como parte de la retroalimentación constante.

\section{FINANCIAMIENTO}

No monetario.

\section{AGRADECIMIENTO}

A la Universidad Regional Autónoma de los Andes, Quevedo, Ecuador y la Universidad César Vallejo, Trujillo, Perú; por motivar el desarrollo de la investigación.

\section{REFERENCIAS CONSULTADAS}

Abreu, O., Gallegos, M., Jácome, J. y Martínez, R. (2017). La Didáctica: Epistemología y Definición en la Facultad de Ciencias Administrativas y Económicas de la Universidad Técnica del Norte del Ecuador [Didactics: Epistemology and Definition in the Faculty of Administrative and Economic Sciences of the Technical University of the North of Ecuador]. Formación Universitaria, 10(3), pp. 81-92.

Area, E. (2014). ¿Qué es el Conectivismo?: Teoría del Aprendizaje Para la Era Digital. [Mensaje en Blog] [What is Connectivism ?: Learning Theory for the Digital Age]. Recuperado de https://n9.cl/au0y2

Bates, A. (2019). Enseñar en la Era Digital [Teach in the Digital Age]. Recuperado de https://n9.cl/1gowm

Buitrago, B. (2008). Didáctica: acontecimiento vivo en el aula [Didáctica: acontecimiento vivo en el aula]. Revista Científica Guillermo de Ockham, 6(2), pp. 55-67.

Correa, A., Di Petta, E. y Frugoni, V. (2011). Interoperabilidad en los Sistemas de Información [Interoperability in Information Systems]. (Tesis de pregrado). Universidad de la Republica, Montevideo, Uruguay. Recuperado de https://n9.cl/915c9 


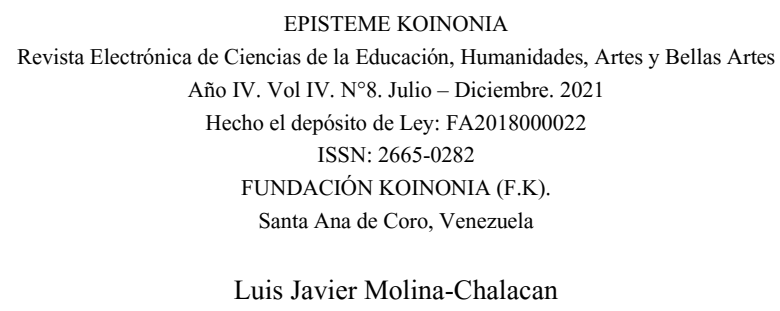

Didáctica. En Diccionario etimológico dechile.net [In etymological Dictionary dechile.net]. Recuperado de https://n9.cl/v0gzt

Downes, S. (2014). El conectivismo como teoría del aprendizaje [Connectivism as a learning theory]. [Mensaje en Blog]. Recuperado de https://n9.cl/trrci7

Dueñas, D., Gómez, A., Toscana, R. y Caro, M. (2017). Sinopsis de metodologias y modelos de Softwer Educativo [Synopsis of methodologies and models of Softwer Educativo]. Acta Scientiae Informaticae, 1(1), 70-74.

Gómez, L. (2007). Interoperabilidad en los Sistemas de Información Documental (SID) [Interoperability in Document Information Systems (SID)]: La información debe fluir. Codice, 3(1), 23-39.

González, B. (2013). El debate sobre la interoperabilidad informática en el derecho de autor comunitario [The debate on computer interoperability in Community copyright]. (Tesis doctoral). Universidad Santiago de Compostela, Santiago de Compostela, España.

González, P. (2017). Conectivismo: La teoría de aprendizaje de la era digital [Connectivism: The Learning Theory of the Digital Age]. Recuperado de https://n9.cl/x0spl

Gutiérrez, L. (2012). Conectivismo como teoría de aprendizaje. Conceptos, ideas y posibles limitaciones [Conectivismo como teoría de aprendizaje. Conceptos, ideas y posibles limitaciones]. Revista Educación y Tecnología, 1, 111-122.

Manso, M., Wachowicz, M., Bernabé, M., Sánchez, A. y Rodríguez, A. (2008). Modelo de Interoperabilidad Basado en Metadatos (MIBM) [Metadata Based Interoperability Model (MIBM)]. Recuperado de https://n9.cl/3r11w

Martinenco, R., Martín, R. y García, L. (2021). Ecologías de aprendizaje en educación secundaria: TIC y aprendizaje informal [Learning ecologies in secondary education: ICT and informal learning Revista Tecnología, Ciencia y Educación, (18), 77-97. DOI https://doi.org/10.51302/tce.2021.571

Martínez, A. y Lara, P. (2006). Interoperabilidad de los contenidos en las plataformas de e-learning: normalización, bibliotecas digitales y gestión del conocimiento [Interoperability of content in e-learning platforms: standardization, digital libraries and knowledge management]. Revista de Universidad y Sociedad del Conocimiento, 3(2).

Nieto, M. (2009). El diseño instruccional de quinta generación basado en la teoría conectivista [Fifth generation instructional design based on connectivist theory]. Recuperado de https://n9.cl/npnf6 
Nogales, P. (2019). Procedimiento para desarrollar la interoperabilidad en proyectos de desarrollo de Sistemas de Gestión Empresarial en Cuba [Procedure to develop interoperability in projects for the development of Business Management Systems in Cuba]. (Tesis de maestría). Universidad de las Ciencias Informáticas, La Habana, Cuba. Recuperado de https://n9.cl/y14i9

Open Assessment Technologies (2019). Interoperabilidad: El eslabón perdido de la tecnología educativa [Interoperability: The Missing Link in Educational Technology]. Recuperado de https://n9.cl/5jf2u

Organización Mundial de la Salud/Organización Panamericana de la Salud. (2016). Revisión de estándares de interoperabilidad para la eSalud en Latinoamérica y el Caribe [Review of interoperability standards for eHealth in Latin America and the Caribbean]. Recuperado de https://n9.cl/uvlhh

Ortiz, A. y Martínez, A. (2017). Sistema de aprendizaje ubicuo en ambientes virtuales [Ubiquitous learning system in virtual environments]. Revista Cubana de Educación Superior, 36(2), 27-40.

Siemens, G. (2004). Conectivismo: Una teoría de aprendizaje para la era digital [Connectivism: A Learning Theory for the Digital Age]. (Trad. Diego Fonseca, 2007). Recuperado de https://n9.cl/c6n5

Silva, D. (2019). Las TIC en la didáctica para la gestión del conocimiento, desde la perspectiva del docente universitario [ICT in didactics for knowledge management, from the perspective of the university teacher]. Dialéctica, Revista de Investigación Educativa, 2.

Sito, L. (2019). Didáctica Universitaria [University Didactics]. Recuperado de https://n9.cl/p79fb

Sunkel, G., Trucco, D. y Espejo, A. (2013). La integración de las tecnologías digitales en las escuelas de América Latina y el Caribe Una mirada multidimensional [The integration of digital technologies in schools in Latin America and the Caribbean A multidimensional view]. Recuperado de https://n9.cl/7fbhi

Vidal, M., Gómez, F. y Ruiz, A. (2010). Software educativo [Educational software]. Educación Médica Superior, 24(1), 97-110.

Vidal, M., Rodríguez, R. y Martínez, G. (2014). Sistemas de gestión del aprendizaje [Learning management systems]. Educación Médica Superior, 28(3), 603-615. 
EPISTEME KOINONIA

Revista Electrónica de Ciencias de la Educación, Humanidades, Artes y Bellas Artes

Año IV. Vol IV. N8. Julio - Diciembre. 2021

Hecho el depósito de Ley: FA2018000022

ISSN: 2665-0282

FUNDACIÓN KOINONIA (F.K)

Santa Ana de Coro, Venezuela

Luis Javier Molina-Chalacan

(02021 por los autores. Este artículo es de acceso abierto y distribuido según los términos y condiciones de la licenciaCreative Commons Atribución-NoComercial-Compartirlgual 4.0 Internacional (CC BY-NC-SA 4.0) (https://creativecommons.org/licenses/by-nc-sa/4.0/). 